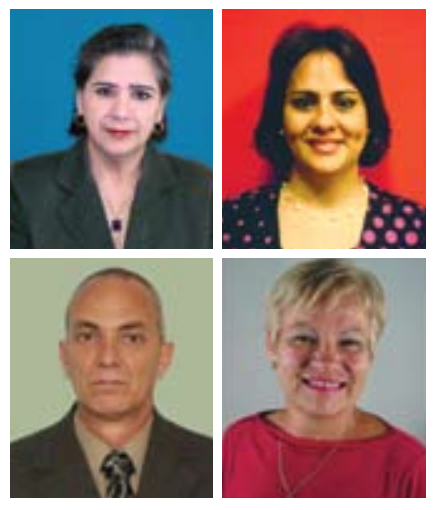

\title{
LA FORMACIÓN CONTINUA ACADÉMICA DE DOCENTES A TRAVÉS DE LA PRÁCTICA REFLEXIVA Y DEL TRABAJO COLABORATIVO EN LA UNIVERSIDAD APEC
}

\author{
Dalma Cruz, lleana Miyar, Jorge García y María de los Ángeles Legañoa *
}

\begin{abstract}
Resumen
Se presenta una

investigación sobre la

formación continua de

docentes en ejercicio en la

Universidad APEC durante

el período 2003-2010. Se

elaboró una estrategia de

gestión que se dinamiza

por la interrelación entre la

formación continua reflexiva

y la formación colaborativa

virtual. Se basa en desarrollar

una formación postgraduada

en ciencias pedagógicas,

junto a supervisiones a

clases y actividades de

sistematización de la

práctica docente, además

de discusiones en foros

virtuales institucionales

y trabajo colaborativo en

redes docentes virtuales.

Esta investigación ofrece

un modelo y una estrategia

para gestionar la formación

continua de docentes en

ejercicio.
\end{abstract}

\section{Palabras clave}

formación del profesorado,

práctica reflexiva,

aprendizaje colaborativo,

redes docentes

\section{Introducción}

La Universidad APEC (UNAPEC), en la búsqueda de la pertinencia, trazó como una de sus estrategias de desarrollo la gestión de la formación continua académica de docentes en ejercicio, a través de las relaciones dialécticas entre formación reflexiva y la formación colaborativa en comunidades virtuales. Para este fin, UNAPEC elaboró una estrategia que constituye un patrón o modelo de decisiones, inspirado en una visión proactiva, con premisas y requisitos. Así, a partir de las regularidades de la gestión universitaria, la estrategia permitió definir y lograr objetivos trascendentes a largo plazo y asignar recursos, posibilitando alcanzar la visión para desarrollar la misión.

La estrategia para una formación continua académica de los docentes universitarios en ejercicio, caracterizada por una formación reflexiva y una formación colaborativa en comunidades virtuales, contribuye a que el claustro cuente con las competencias académicas para enfrentar los retos que la sociedad del conocimiento impone a las universidades.

\section{Fundamentos Teóricos}

La estrategia propuesta en su enfoque sistémico se estructuró en tres procesos: la formación, la informatización y la dirección del procesodocenteeducativo.Separtiódeconsiderar que desde las relaciones entre estos procesos, las relaciones entre los actores $y$. las de éstos con los procesos, la universidad asegura la formación continua académica de los docentes en ejercicio. Es a través de las relaciones entre los procesos (formación, informatización, dirección del proceso docente educativo), de las relaciones de los actores y de unos con otros, que se gestiona y fomenta la formación continua académica. A partir de esas relaciones se logra dirigir las acciones para que a través de la reconversión docente y la transposición de saberes, como resultado de una práctica reflexiva y un trabajo colaborativo en comunidades virtuales, se potencie la profesionalización del docente y la consolidación de una identidad grupal virtual de los docentes.

La formación es entendida como el proceso que se desarrolla a través de las relaciones sociales establecidas entre sus actores, con el propósito de educar e instruir. Quedan im-

\footnotetext{
* Dalma Cruz es Magíster en Gestión Universitaria por la Universidad de Alcalá de Henares, España, Doctora en Ciencias Pedagógicas y Vicerrectora de la Escuela de Graduados de la Universidad APEC.
}

Ileana Miyar es Doctora en Ciencias Pedagógicas por la Universidad de Camagüey de Cuba y Directora de Asuntos Docentes de esa Institución.

Jorge García es Doctor en Ciencias Pedagógicas por la Universidad de Camagüey, donde tiene el cargo de Vicedecano de Investigaciones de la Facultad de Electromecánica.

María de los Ángeles Legañoa es Doctora en Ciencias Pedagógicas por la Universidad de Camagüey, donde trabaja como investigadora del Centro de Estudios de Ciencias de la Educación "Enrique José Varona". 
plícitas las tres funciones del proceso: la educativa, la instructiva y la desarrolladora. La función instructiva puede verse asociada con el acceso del docente a los diferentes saberes acumulados y sistematizados por la humanidad. La función educativa es aquella asociada a la formación de sentimientos, convicciones, valores, voluntad y espíritu de los docentes en el contexto social e institucional. La función desarrolladora apunta al desarrollo de capacidades investigativas, con lo que no sólo se compromete al docente con el propio proceso, sino que desarrolla sus potencialidades transformadoras.

\section{La informatización es vista como:}

- El amplio acceso, multiplicidad y replicabilidad de los diversos recursos de las universidades.

- El desarrollo de competencias necesarias para la gestión del conocimiento, a través de comunidades virtuales.

- La interacción que supera las dimensiones y la rigidez del tiempo y el espacio, lo cual representa transformar la práctica educativa.

La dirección del proceso docente educativo es vista como:

- El proceso de diseño y mantenimiento de un ambiente en el que las personas, trabajando en grupos, alcancen con eficiencia metas seleccionadas.

- La planificación, organización, la regulación (dirección) y el control del proceso docente educativo.

- La coordinación para la toma de decisiones al nivel más funcional del proceso, de acuerdo con lo decidido en planos más estratégicos de naturaleza institucional, de mando, sobre la base de la política y la filosofía de dicha institución.

- La formación de los profesores como condición necesaria para su buena actuación.

Para el desarrollo de la estrategia, se propuso un sistema de rasgos de las cualidades de formación reflexiva y formación colaborativa en comunidades virtuales, a través de los cuales se desarrolla la formación académica continua. De acuerdo con los estudios realizados, seasumencomorasgos delaformación reflexiva: laformación de postgrado, la sistematización de la experiencia educativa y la supervisión.

La formación de postgrado es vista como:

- La articulación armónica de la formación, el desarrollo, la investigación y la innovación.
- El desarrollo de procesos continuos de creación, difusión, transferencia, adaptación y aplicación de conocimiento.

- La promoción de la colaboración institucional e interinstitucional de carácter nacional, regional e internacional.

- La adopción de formas organizativas flexibles, a través de modalidades a tiempo parcial o completo, y de comparecencia presencial o semi-presencial.

- La atención a las necesidades personales, el trabajo colaborativo y la integración en redes.

- La estructuración sistémica de la superación profesional y la formación académica.

- Lasuperación profesional como expresión de una formación continua y de la actualización sistemática.

- La formación académica como expresión del desarrollo de alta competencia profesional e investigativa.

La sistematización de la experiencia educativa es entendida como el proceso de reflexión crítica que tiene el propósito de provocar procesos de aprendizaje. Se orienta a describir y entender lo que sucedió en una experiencia y explicar por qué se obtuvieron esos resultados, y como se deberá accionar para mejorar en el futuro. Además, se basa en la idea de ordenar lo disperso o desordenado (prácticas, conocimientos, ideas, datos, percepciones, opiniones, etc.). La supervisión es un proceso único e integral, cuya acción va dirigida a ayudar, apoyar el compartir y a contribuir para que el docente en ejercicio progrese en su propia práctica. Además, es una ac-

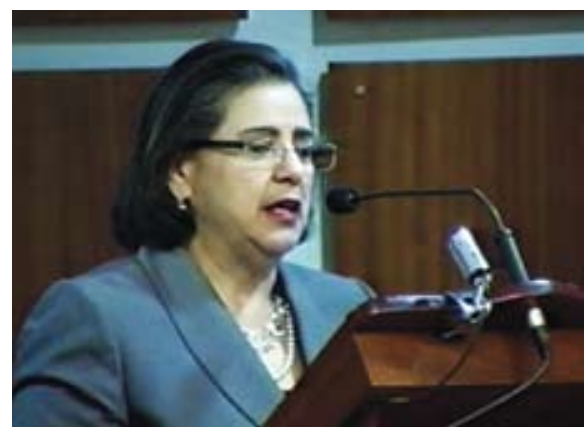
ción permanente orientada al mejoramiento de los resultados del proceso enseñanza-aprendizaje, atañe a todas las actividades y a todas los actores que concurren en el proceso educativo y se realiza mediante el trabajo de pares.

Se asumen como rasgos de la cualidad formacióncolaborativaencomunidades virtuales: la formación posgraduada, la discusión en foros virtuales institucionales y el trabajo colaborativo en redes docentes.

La discusión en foros virtuales institucionales permite informar, discutir o reflexionar sobre aspectos generales o más específicos de la gestión académica universitaria. Las plataformas como espacios virtuales específicos para la formación no presencial han incorporado y dado contenido de uso a tal herramienta como un mecanismo abierto a la colaboración, sobre todo de carácter horizontal, entre los 
gestores educativos, muy especialmente en la parte de la acción del docente. El foro tiene una alta potencialidad formativa en procesos de reflexión compartida sobre aquellos temas que son objeto de estudio, potenciando así, no sólo apropiarse de las orientaciones emanadas por la dirección de la institución, sino también de las interpretaciones o puntos de vista que otros pudieran aportar. La discusión en foros virtuales institucionales tiene las siguientes finalidades:

- El foro como un lugar de encuentro para abordar las cuestiones de la gestión académica a nivel institucional.

- El foro como espacio de reflexión compartida, para abordar temáticas específicas de la gestión académica, a nivel institucional.

- El foro como espacio para la colaboración en actividades compartidas emanadas de la gestión académica a nivel institucional.

El trabajo colaborativo en redes docentes es un proceso que posibilita a los profesores la valoración de su actividad, la experimentación de nuevas formas de trabajo y el intercambio con otros colegas. Propicia la construcción de un saber pedagógico basado en la propia experiencia y en la experiencia de los demás. Las redes proporcionan estructuras organizativasquepermitenalosgrupos deprofesoresreunirse para hablar de su trabajo, aprender de otros y conducir temas curriculares y estructurales. En este se lleva a cabo:

- El intercambio de nuevos conocimientos surgidos tanto de la investigación básica y aplicada como de la práctica profesional. Ejemplos de ello son: las revistas y conferencias electrónicas y las listas de discusión.
- La colaboración para mejorar las aptitudes y resolver problemas. Este tipo de colaboración es requerida más allá que, un simple intercambio de información. Debe ser un intercambio de ideas, compartir experiencias y discutir soluciones de las dificultades que se dan entre profesores; por ejemplo, pueden dar lugar, a través de redes, a grupos de trabajo mucho más amplios y diversos.

- La colaboración para crear nuevo conocimiento. Distintos profesores trabajan juntos durante largos períodos para lograr metas compartidas. Ejemplos típicos de esta categoría son: el desarrollo de materiales curriculares por un grupo de profesores, la escritura de un artículo en común, la realización de investigaciones y proyectos de innovación por equipos de profesores de distintos centros, y el intercambio de productos de las actividades.

- Para su implementación, se concibió la estrategia de la participación de tres actores principales: rectorado, decanato, y el docente. Los actores en el desarrollo de la estrategia, como se ha expresado, juegan un papel esencial a través de las relaciones entre cada uno en el proceso y la función asignada para el cumplimiento de los objetivos previstos. Las relaciones entre los actores están sustentadas, además de las exigencias pedagógicas, didácticas, psicológicas, etc. que forman parte de los procesos involucrados, por los rasgos de las cualidades de la gestión.

De forma sintética, el modelo de esta estratégia se presenta en la gráfica 1 .

Gráfica 1. Modelo de la estrategia de formación continua de los docentes






\section{La gestión}

La estrategia de gestión se desarrolló en cuatro fases: diagnóstico inicial, planificación, ejecución y control. A continuación se explica cómo las acciones desarrolladas en cada una de ellas; están dirigidas a fortalecer la integración de las relaciones entre la formación reflexiva y la formación colaborativa, en comunidades virtuales.

\section{a) Diagnóstico Inicial}

El diagnóstico inicial, como parte de la gestión institucional, se orientó hacia la evaluación de los factores externos e internos que condicionaron la determinación de los objetivos estratégicos que tenían como centro la determinación de las necesidades de formación continua académica de los docentes, así como el diagnóstico de las fortalezas, debilidades, oportunidades y amenazas relacionadas con el mismo proceso de formación.

La determinación de las necesidades académicas y formativas de los docentes se elaboró a partir del modelo educativo en el cual se expresa: "El profesor de UNAPEC debe estar comprometido con la misión de la universidad, siendo ejemplo para los alumnos en cuanto a las actitudes y competencias que éstos deben poseer. Para ello se requiere: estar actualizado en los conocimientos de su especialidad profesional, poseer un conocimiento profesional como docente, ser capaz de realizar actividades investigativas, de extensión y de gestión. Asimismo, ha de tener una concepción internacional del currículum, de la práctica profesional y académica, así como de los resultados de los cuales son responsables en su quehacer profesional docente. El docente debe ser capaz de diseñar y propiciar ambientes de aprendizaje que promuevan el encuentro con el conocimiento, la integración social y la capacidad de adaptación a la sociedad cambiante que identifica el tercer milenio. El docente de UNAPEC debe ser capaz de dirigir a sus estudiantes hacia interrelaciones que propicien la actividad, y por ende, la posibilidad de reconstruir los contenidos discutidos en el aula, evitando la repetición y evaluando la incorporación de esos saberes en su estructura cognoscitiva".

\section{b) Planificación}

En esta fase, desde la gestión institucional se realizaron diferentes acciones:

Se elaboraron los instrumentos para la realización del diagnóstico específico con el propósito de caracterizar el estado de los docentes en cuanto a su formación pedagógica y sus necesidades formativas.

Se identificaron los objetivos estratégicos en función de los resultados del diagnóstico. Estos fueron:
Objetivo estratégico general: Alcanzar niveles cualitativamente superiores en la formación continua académica de los docentes en ejercicio de la Universidad APEC, a fin de propiciar un desarrollo del proceso de formación profesional, sustentado en la práctica reflexiva y el trabajo colaborativo en comunidades virtuales. De este objetivo se derivaron 3 estrategias específicas, cuyos objetivos fueron:

- Estrategia 1. Desarrollo de la formación académica de los docentes en ejercicio: Desarrollar un proceso de formación académica de los docentes en ejercicio.

- Estrategia 2. Desarrollo de la práctica reflexiva: Establecer los mecanismos de acción que garanticen la continuidad de la formación académica, la supervisión de las actividades docentes y la sistematización de las experiencias educativas en cada una de las áreas.

- Estrategia 3. Desarrollo del trabajo colaborativo en comunidades docentes virtuales: Establecer los mecanismos que garanticen la continuidad de la formación académica sustentada en el trabajo colaborativo docente, a través del establecimientos de foros virtuales institucionales y las redes colaborativas de docentes.

Se hizo la proyección del plan de acciones basado en el diseño de la definición de nuevas estrategias. Como base o premisa para el desarrollo de éstas, se tomó el programa de postgrado en Ciencias Pedagógicas, producto de un proyecto colaborativo internacional convenido por la Vicerrectoría de Internacionalización y Cooperación con la Universidad de Camagüey, Cuba, denominado "Desarrollo Profesional Docente". Este programa compuesto por tres fases de capacitación pedagógica en las ciencias particulares en que imparten docencia los profesores, la Maestría en Ciencias de la Educación y el doctorado en Ciencias Pedagógicas. Se planificó capacitar el $75 \%$ de los docentes, 50 de ellos continuarían la maestría y 20 el doctorado.

La coordinación de la planificación, implementación y evaluación de esta formación estuvo a cargo del Departamento de Control y Formación Docente.

Para la capacitación didáctica especializada se organizaron cohortes por áreas profesionales. Para determinar las cohortes de capacitación, se partió de los siguientes criterios: los participantes tenían que pertenecer a una misma área profesional, había que capacitar, al menos, el 75\% de los docentes de cada área y cada cohorte podía tener como máximo, 40 integrantes. De esta manera, se constituyeron dos grupos de 20 alumnos cada una de las áreas. 
La aplicación de estos criterios permitió establecer las cohortes de capacitación, quedando de la forma siguiente en la Tabla 1:

Tabla 1. Grupos participantes

en el Postgrado en Ciencias Pedagógicas

\begin{tabular}{lccc}
\hline Área & $\begin{array}{c}\text { Facultades/ } \\
\text { Departamentos }\end{array}$ & $\begin{array}{c}75 \% \\
\text { del total } \\
\text { de profesores }\end{array}$ & $\begin{array}{c}\text { No. de } \\
\text { Cohortes }\end{array}$ \\
\hline $\begin{array}{l}\text { Ciencias } \\
\text { Empresariales } \\
\text { y Económicas }\end{array}$ & $\begin{array}{c}\text { Administración } \\
\text { Contabilidad } \\
\text { Mercadeo Turismo } \\
\text { Esc. Graduados }\end{array}$ & 128 & 3 \\
$\begin{array}{l}\text { Ciencias de } \\
\text { la Ingeniería }\end{array}$ & $\begin{array}{c}\text { Ingenieríay } \\
\text { Tecnología }\end{array}$ & 34 & 1 \\
$\begin{array}{l}\text { Ciencias } \\
\text { Informáticas }\end{array}$ & $\begin{array}{l}\text { Informática } \\
\text { Ciencias }\end{array}$ & 33 & 1 \\
Sociales & Sociales & 40 & 1 \\
Matemática & Matemática & 37 & 1 \\
Ciencias del & Arte & 78 & 2 \\
Diseñoy la \\
Comunicación
\end{tabular}

Además, se planificaron cursos en Tecnología Educativa con la intención de preparar a los docentes en las TIC para la Educación; estos comprendían la formación en redes.

Se implementó una plataforma tecnológica para la capacitación a los docentes, así como para los foros virtuales institucionales y se diseñó un centro de apoyo a la docencia para dar soporte a los docentes en Tecnología Educativa.

Se diseñaron los cursos de capacitación, según una modalidad híbrida, los instrumentos para la realización de la práctica reflexiva y el trabajo colaborativo en comunidades virtuales.

\section{c) Ejecución}

La primera estrategia que se implementó fue la del Desarrollo de la formación académica de los docentes en ejercicio. La primera fase de la capacitación estaba constituida por un programa de postgrado dirigido a la capacitación didáctica especializada en sus áreas profesionales, estructurada en dos módulos, cada uno compuesto de cuatro asignaturas. El primer módulo, de formación pedagógicageneral yel segundo, dedicado a la metodología de la enseñanza de las ciencias particulares. La capacitación comenzó en agosto del 2003, con dos cohortes, una de Ciencias Empresariales y otra, de Ciencias de las Ingenierías. En la actualidad se han desarrollado 10 cohortes de capacitación, para un total de 360 capacitados.

La concepción de la capacitación por cohortes de áreas particulares, dirigida a la dinamización del profesor hacia propuestas de innovación, a través del cuestionamiento inicial de su pensamiento docente espontáneo y su praxis, posibilitó que los docentes de una misma disciplina se conocieran, trabajaran juntos en sus asignaturas, intercambiaran sus experiencias y se propiciaran los procesos de transposición de saberes, adquiriendo una identidad grupal producto del trabajo en equipo.

La segunda fase de formación correspondió al desarrollo de la Maestría de Ciencias de la Educación iniciada en mayo del 2004. En ella se matricularon 129 docentes, los cuales se agruparon en 4 cohortes de Maestría y realizaron su proceso de formación escalonadamente. Cada docente desarrolló una investigación relacionada con un problema diagnosticado por los directivos de la universidad, tomando en cuenta las necesidades de desarrollo de la institución en vías a la excelencia académica. En la actualidad, 102 docentes han culminado sus investigaciones educativas y han defendido sus tesis de Maestría, duplicando el número esperado en un principio ante la demanda y aceptación del proceso por los profesores de la Institución. Esta fase del proceso proporcionó una nueva cualidad al trabajo en equipo, pues en esta, los profesores se unieron para implicarse en procesos de investigación educativa. Las principales dificultades se presentaron por la inexperiencia de los docentes en la realización de investigaciones, las cuales fueron atendidas por las asesorías y el trabajo colaborativo de los compañeros de cohorte.

La tercera fase fue la del doctorado en Ciencias Pedagógicas. Este inició en octubre del 2006. Fueron aceptados a cursar el Doctorado, en una modalidad tutorial, un total de 16 docentes. De ellos, hay 8 que han culminado sus investigaciones y han obtenido el título de Doctor en Ciencias Pedagógicas. En el proceso de formación científica y desarrollo de las investigaciones, el trabajo en equipo desempeñó un rol esencial, debido a que todos los aspirantes exhibían diferentes niveles de competencia investigativa. El desarrollo de seminarios de tesis, celebrados periódicamente y donde participaban todos los aspirantes aportando sus conocimientos a cada investigación, fue una experiencia muy valiosa porque permitió la creación del nuevo instrumento para defender la tesis doctoral. También, fue un elemento muy importante la contribución de cada 
aspirante que alcanzaba el grado a la formación de los que aún no la habían alcanzado. Todas estas evidencias demostraron la validez del trabajo colaborativo docente.

La segunda estrategia, relacionada con el desarrollo de la práctica reflexiva, comenzó a implementarse para dar continuidad a la capacitación y convertir la formación en un proceso continuo. En la misma, se adiestraron a grupos de docentes para la elaboración de portafolios que posibilitaran sistematizar sus prácticas docentes y se implementó, además, un proceso de supervisión, tanto por directivos como por pares, para valorar la praxis de los docentes y ofrecer la ayuda oportuna para su perfeccionamiento. El Centro de Apoyo a la Docencia ha desempeñado un rol importante en la misma, tanto en la asesoría a los docentes como en el control del proceso.

La tercera estrategia, dirigida al desarrollo de comunidades virtuales docentes, se relaciona estrechamente con las estrategias anteriores, dado que en ella se da un aprendizaje social colaborativo que se sustenta en el desarrollo profesional de cada docente. Para la implementación de la misma, se han desarrollado foros institucionales, en los cuales se han debatido temáticas relacionadas con la política de innovación de la universidad. Ejemplo de esos foros fueron los desarrollados para el diseño de sílabos, así como los relacionados con las guías didácticas para la modalidad semipresencial.

En la actualidad, se trabaja por la implementación de redes docentes virtuales para desarrollar la intercreatividad, con el objetivo de motivar a los docentes a realizar cosas y a resolver problemas juntos. Este proceso no solo refuerza la capacidad de transferir información entre los docentes, sino que va mucho más allá, pues le asigna un valor estratégico al proceso social de intercambio y a la construcción colectiva del saber.

\section{d) Control}

Esta fase tuvo como objetivo valorar la marcha y la aplicación de la estrategia, además, establecer las correcciones y adecuaciones necesarias para su perfeccionamiento, permitiendo la retroalimentación permanente.

Las acciones fundamentales que se desarrollaron fueron:

- Análisis sistemático sobre la calidad de la apropiación, por parte de los docentes, de los contenidos tratados a través de los diversos cursos.

- Aplicación de instrumentos que permitieron obtener criterios valorativos acerca de la calidad de las figuras del postgrado vinculadas a la estrategia, así como el resto de las actividades.
- Valoración en torno a la forma en la que los docentes incorporaron lo aprendido a la práctica pedagógica.

- Valoración en torno al aporte del trabajo en equipo a los docentes.

- Realización de los ajustes necesarios para el perfeccionamiento de la estrategia.

\section{Conclusiones}

La estrategia propuesta, tomando como base las regularidades expuestas, sirve de guía a los directivos y profesores que intervienen en el proceso de formación continua académica, pudiéndose utilizar como vía para incrementar la formación académica de los profesionales en ejercicio mediante el desarrollo de una práctica reflexiva y el trabajo colaborativo en comunidades virtuales.

La implementación de la estrategia, hasta la fecha, ha demostrado la necesidad del trabajo colaborativo entre docentes, como sustento de la profesionalidad colectiva, la cual constituye un estadio superior en el desarrollo profesional de los docentes.

\section{Referencias bibliográficas}

Álvarez, C. (1998).Pedagogía como ciencia. La Habana: Editorial de la Universidad de La Habana.

Amaya, G. (1996). La formación permanente de docentes: un proyecto que debemos pensar y construir. Bogotá: Educación y cultura.

Cabero, J. (2002). Tecnología Educativa. Diseño y utilización de medios en la enseñanza. Barcelona: Paidós.

Davini, M. C. (2005). La formación docente en cuestión: política ypedagogía. Buenos Aires: Paidos.

Gadino, A. (2005). La construcción del pensamiento reflexivo. Santa Fé: Homo - Sapiens.

Núñez N. \& Palacios, P. (2002). Modelo teórico para un programa de capacitación continua de docentes en servicio en educación básica. Tesis doctoral no publicada. Universidad de Lambayeque, Perú.

Perrenoud, P. (2002). Políticas de incentivos sobre el profesorado universitario:Situación actual y propuestas de mejora. Ginebra: Université de Genève.

Salinas J. (s. f.) Redes y desarrollo profesional del docente: Entre el dato serendipitiy el foro de trabajo colaborativo. Extraído en 2007, de www.uib.es

Schön, D. (1998). El profesional reflexivo, cómo piensan los profesionales cuando actúan. Barcelona: Paidos. 